\title{
Neuroglobin-overexpression reduces traumatic brain lesion size in mice
}

\author{
Song Zhao ${ }^{1,2}$, Zhanyang $\mathrm{Yu}^{2}$, Gang Zhao ${ }^{1}$, Changhong Xing ${ }^{2}$, Kazuhide Hayakawa ${ }^{2}$, Michael J Whalen ${ }^{3}$, \\ Josephine M Lok ${ }^{2,3}$, Eng $\mathrm{HLO}^{2}$ and Xiaoying Wang ${ }^{2,4^{*}}$
}

\begin{abstract}
Background: Accumulating evidence has demonstrated that over-expression of Neuroglobin ( $\mathrm{Ngb}$ ) is neuroprotective against hypoxic/ischemic brain injuries. In this study we tested the neuroprotective effects of Ngb over-expression against traumatic brain injury (TBI) in mice.

Results: Both Ngb over-expression transgenic (Ngb-Tg) and wild-type (WT) control mice were subjected to TBI induced by a controlled cortical impact (CCl) device. TBI significantly increased Ngb expression in the brains of both WT and Ngb-Tg mice, but Ngb-Tg mice had significantly higher Ngb protein levels at the pre-injury baseline and post-TBI. Production of oxidative tissue damage biomarker 3NT in the brain was significantly reduced in Ngb-Tg mice compared to WT controls at 6 hours after TBI. The traumatic brain lesion volume was significantly reduced in $\mathrm{Ngb}$ Tg mice compared to WT mice at 3 weeks after TBl; however, there were no significant differences in the recovery of sensorimotor and spatial memory functional deficits between Ngb-Tg and WT control mice for up to 3 weeks after TBI.
\end{abstract}

Conclusion: Ngb over-expression reduced traumatic lesion volume, which might partially be achieved by decreasing oxidative stress.

Keywords: Neuroglobin, Neuroprotection, Controlled cortical impact, Oxidative stress, Traumatic brain injury

\section{Background}

Neuroglobin (Ngb) is a member of the tissue globin family with a high affinity for oxygen, and it is widely expressed in the neurons of vertebrate central and peripheral nervous systems, retina, and endocrine tissues [1-3]. Accumulating evidence from our lab and others has demonstrated that Ngb over-expression is neuroprotective against hypoxic/ischemic brain injuries in cell cultures and stroke animal models, as well as in beta-amyloid-induced neurotoxicity in vitro and Alzheimer's phenotype in mice [4-7]. Although the underlying neuroprotective mechanisms remain largely unknown, experimental studies have suggested that $\mathrm{Ngb}$ may act as an oxygen sensor in regulating the

\footnotetext{
*Correspondence: wangxi@helix.mgh.harvard.edu

${ }^{2}$ Neuroprotection Research Laboratory, Departments of Neurology and

Radiology, Massachusetts General Hospital, Harvard Medical School, Charlestown, MA, USA

${ }^{4}$ Neuroprotection Research Laboratory, Departments of Neurology and Radiology, Massachusetts General Hospital, 149 13th Street, Room 2401, Charlestown, MA 02129, USA

Full list of author information is available at the end of the article
}

signaling pathway, attenuating oxidative stress and preserving mitochondrial function [4,8-11]. Traumatic brain injury is one of the major acute neurological disorders, but the effects of Ngb over-expression on its neurological outcomes remain uninvestigated. Therefore, in this study we tested whether Ngb overexpression is neuroprotective against traumatic brain injury in mice.

\section{Methods}

All animal experiments were performed following protocols approved by the Massachusetts General Hospital Animal Care and Use Committee in compliance with the National Institutes of Health Guide for the Care and Use of Laboratory Animals.

\section{Controlled cortical impact model of traumatic brain injury} The standard controlled cortical impact model of traumatic brain injury was performed as previously described $[12,13]$. Briefly, a right craniotomy was made using a portable drill and $5 \mathrm{~mm}$ trephine over the right parieto-

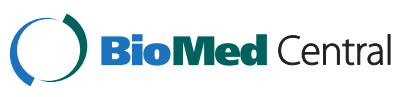


temporal cortex, and the bone flap was removed. Mice were subjected to CCI using a pneumatic cylinder with a 3 -mm flat-tip impounder, velocity $6 \mathrm{~m} / \mathrm{s}$, depth of $0.6 \mathrm{~mm}$, and $150 \mathrm{~ms}$ impact duration. Ngb-Tg mice and WT control mice in C57BL/6 background were ear-tagged and assigned randomly into two equal-sized groups, blinded to the investigators.

\section{Immunohistochemistry}

Immunohistochemistry was performed following the standard method as we previously described [5]. Briefly, at $6 \mathrm{~h}$ after TBI, mice were anesthetized with an overdose isoflurane and were transcardially perfused with chilled $\left(4^{\circ} \mathrm{C}\right) \mathrm{PBS}(\mathrm{pH} 7.4)$. Coronal sections $(12 \mu \mathrm{m})$ on slides were fixed in $100 \%$ cold aceton and blocked in PBS with $3 \%$ bovine serum albumin for $1 \mathrm{~h}$ and incubated at $4^{\circ} \mathrm{C}$ overnight in a PBS solution containing the rabbit anti-Ngb antibody (1:50; Santa Cruz Biotechnology, CA, US) in $0.3 \%$ bovine serum albumin. The sections were then washed and incubated for $1 \mathrm{~h}$ with Rhodamine (TRITC)-AffiniPure Goat Anti-Rabbit IgG $(\mathrm{H}+\mathrm{L})(1: 200$; Jackson ImmunoResearch, PA, US). Signals in perilesion cortex were examined using an Olympus microscope (BX51; Olympus) equipped with rhodamine filter set.

\section{Western blots}

Western blots analysis was performed following the standard method as we previously described [5]. Briefly, injured brain hemisphere tissues were dissected on ice, followed by protein extraction. Protein concentrations in the supernatants were measured with Bradford assay (Bio-Rad, Hercules, CA, USA). Equal amount of protein were separated in a 4-20\% Tris-glycine gel (Invitrogen) (40 $\mu \mathrm{g} /$ lane) and then transferred onto PVDF Membranes. The membranes were incubated with primary antiNgb antibody (1:1000; Biovendor R\&D, NC, USA) and anti- $\beta$-actin antibody $\left(1: 10000\right.$; Sigma) at $4^{\circ} \mathrm{C}$ overnight. Then the membranes were incubated with horseradish peroxidase-linked rabbit polyclonal anti-Chicken IgY (1:2000, Abcam, MA, USA) and anti-mouse IgG (1:10000, GE Healthcare, UK), respectively for $1 \mathrm{hr}$ at room temperature, developed by enhanced chemiluminescent (Pierce, Rockford, IL, USA). Densitometric analysis was performed for quantitation with Image J software.

\section{Slot-immunoblotting analysis}

3-nitrotyrosine (3NT) production in brain tissues after TBI was assessed with slot-immunoblotting analysis followed by a standard method described by others, with minor modifications [14,15]. Briefly, at $6 \mathrm{~h}$ after TBI, mice were transcardially perfused with chilled $\left(4^{\circ} \mathrm{C}\right) \mathrm{PBS}$ $(\mathrm{pH} 7.4)$, and the brains were carefully removed and placed in a cooled $\left(6^{\circ} \mathrm{C}\right)$ brain matrix. A $3 \mathrm{~mm}$ thick slice was cut in coronal orientation and separated in quadrants. The right-upper (traumatized) region was collected, frozen in liquid nitrogen and stored at $-80^{\circ} \mathrm{C}$ until further processing. To measure 3-nitrotyrosine (3NT) production, an aliquot of each ipsilateral brain sample $(1 \mu \mathrm{g})$ was diluted with $200 \mu \mathrm{l}$ of tris-buffered saline (TBS) and transferred to a protran nitrocellulose membrane (Whatman Inc., ME, US) by a Minifold I vacuum slot blot apparatus (Whatman Inc., ME, US). After the samples were loaded into the slots, they were allowed to filter through the membrane by vacuum. Each slot was then washed with $200 \mu \mathrm{l}$ TBS, which was allowed to filter through the membrane again. The membranes were disassembled from the apparatus and incubated in a TBS blocking solution with 5\% milk for $1 \mathrm{~h}$ at room temperature. Then the membranes were incubated with rabbit polyclonal anti-nitrotyrosine antibody (1:2000; Sigma, MA, US) at $4^{\circ} \mathrm{C}$ overnight. After the primary incubation, the membranes were washed and then incubated again with horseradish peroxidaselinked anti-rabbit IgG (1:2000, Abcam, MA, USA) for $1 \mathrm{~h}$ at room temperature, developed by enhanced chemiluminescent (Pierce, Rockford, IL, US). Densitometric analysis was performed for quantitation with Image J software.

\section{Assessments of sensorimotor functions}

A 10-point neurological severity score (NSS) was used for the assessment of posttraumatic neurological impairment, as previously described [16,17]. The score consists of 10 individual clinical parameters, including tasks on motor function, alertness, and physiological behavior, whereby 1 point is given for failure of one task and no points are given for success. A maximal NSS of 10 points indicates severe neurological dysfunction, with failure at all tasks. The NSS was assessed at 1, 3, 5, 7, 10, 14 and 21 days after TBI. All mice were trained and pretested prior to injury. Vestibulomotor function was assessed using a standard wire-grip test [18]. Mice were placed on a metal wire (45 cm long) suspended $45 \mathrm{~cm}$ above a foam pad and were allowed to traverse the wire for 60 seconds. The latency that a mouse remained on the wire within a 60 second interval was measured, and wire grip scores were quantitated using a 5-point scale. $0=$ fell off the wire within the $30 \mathrm{~s}$ period, 1 = held on in some way for $30 \mathrm{~s}, 2=$ held on with four paws for $\geq 5 \mathrm{~s}$, 3 = held on with four paws and placed tail on wire for $\geq 5 \mathrm{~s}, 4=$ held on with four and move to the end of the wire, $5=$ move to the end of the wire and go down to the ground with four paws The tests were performed in triplicate, and an average value was calculated for each mouse on each day of testing. Baseline assessment was done the day before injury. 


\section{Assessments of spatial memory functions}

The Morris Water Maze task was used to evaluate spatial memory performance from 15 days to 21 days after TBI as previously described [18]. Briefly, performance in the MWM was quantitated by latency to the platform. The apparatus consisted of a white pool (90 cm diameter, $60 \mathrm{~cm}$ deep) filled with water to $29 \mathrm{~cm}$ depth, with several highly visible cues located on the walls of each of the four quadrants. Water temperature was maintained between $21^{\circ} \mathrm{C}$ and $25^{\circ} \mathrm{C}$. A clear Plexiglass goal platform $5 \mathrm{~cm}$ in diameter was positioned $0.5 \mathrm{~cm}$ below the water surface approximately $15 \mathrm{~cm}$ from the southwest wall. Each mouse was subjected to a series of four trials per day. For each trial, mice were randomized to one of four starting locations (north, south, east, or west) and placed in the pool facing the wall. Mice were given a maximum of 60 seconds to find the submerged platform. If the mouse failed to reach the platform by 60 seconds, it was placed on the platform by the experimenter and allowed to remain there for $15 \mathrm{sec}-$ onds. Mice were placed in a warming chamber for at least 4 minutes between trials. To control for possible differences in visual acuity or sensorimotor function between groups, two trials were performed using a visible platform raised $1 \mathrm{~cm}$ above the surface of the water. Performance in the MWM was quantitated by latency to find the platform.

\section{Quantitation of traumatic lesion volume}

Quantitation of traumatic lesion volume was performed by following a standard method as previously described [5]. At 3 weeks after TBI, mice were sacrificed and transcardially perfused with chilled $\left(4^{\circ} \mathrm{C}\right) \mathrm{PBS}, \mathrm{pH} 7.4$, followed by $4 \%$ paraformaldehyde in $0.1 \mathrm{M}$ PBS. Frozen brains were sectioned with microtome into $20-\mu \mathrm{m}$-thick coronal slices. Every 20th section was mounted onto a glass slide and stained with hematoxylin and eosin. Histological lesion areas were quantified with a standard computer-assisted image analysis program (Image J), and the lesion areas were then integrated to obtain total lesion volumes in cubic millimeters.

\section{Statistical analysis}

All data were expressed as mean \pm SD. Sensorimotor and MWM were analyzed by two-factor repeatedmeasures analysis of variance (group $\times$ time). Slotimmunobloting and western blot data were analyzed by one-way ANOVA. Lesion volumes were analyzed by t-test. For all comparisons, $\mathrm{P}<0.05$ was considered significant.

\section{Results and discussion}

Increasing evidence has demonstrated that Ngb plays an important role in neuroprotection against hypoxic/ ischemic brain injury, such as stroke and other related neurological disorders $[5,19,20]$. Our previous studies showed that Ngb overexpression reduces tissue infarction and markers of oxidative stress in a focal stroke mouse model [5]. Recent reports by others have shown that Ngb gene expression was increased after TBI, but its effects on outcomes were not examined [21,22]. In this short study we tested our hypothesis that Ngb overexpression may protect against traumatic brain injury in mice. We performed three sets of experiments to examine (1) changes in brain Ngb protein expression after TBI, (2) the effects of Ngb over-expression in a mechanistic endpoint-oxidative tissue damage, and (3) the neurological outcomes of neurobehavioral deficits and traumatic lesion size at three weeks after TBI. Both Ngb-Tg and WT control mice were examined and compared.

In the first set of experiments, we found that Ngb protein level in the injured brains were significantly increased in both $\mathrm{Ngb}-\mathrm{Tg}$ and WT mice compared to their sham controls at $6 \mathrm{~h}$ after TBI. This examination by immunohistochemistry (Figure 1A) and western blot analysis (Figure 1B) was consistent with a report that showed a transient increase of brain Ngb expression with peak at $6 \mathrm{~h}$ after TBI in rats. That study also showed Ngb over-expression decreased mechanical injury-induced neuron death [23]. In our present study, quantification of western blot analysis showed that the basal levels of Ngb protein expression (149\% of WT sham control mice) and the CCI-induced increase of Ngb protein levels at $6 \mathrm{~h}$ after TBI (196\% of WT sham control mice) were significantly higher in Ngb-Tg mouse brains compared to WT TBI controls (147\% of WT sham control mice) (Figure1C). While this difference suggested that TBI induces Ngb protein expression, the basal and TBI-induced Ngb protein levels were still significantly higher in Ngb-Tg mouse brains, validating the possible causality between the different brain Ngb protein levels in association with different neurological outcomes after TBI of Ngb-Tg and WT control mice.

Previous studies have demonstrated that Ngb overexpression can reduce hypoxia/ischemia-induced oxidative cell damage in cultured neurons and focal cerebral ischemia in mice $[5,19]$. In the second set of experiments, we tested and compared a common oxidative tissue damage biomarker, 3NT production, in TBI-injured brains of Ngb-Tg and WT control mice. We found that there was a significant reduction of 3NT level at $6 \mathrm{~h}$ after TBI in Ngb-Tg mouse brains as compared to WT controls (Figure 2), indicating TBI-induced oxidative tissue damage may be diminished by Ngb over-expression.

In the last set of experiments, we examined neurological outcomes of neurobehavioral deficits for up to three weeks after TBI and traumatic brain lesion size at 


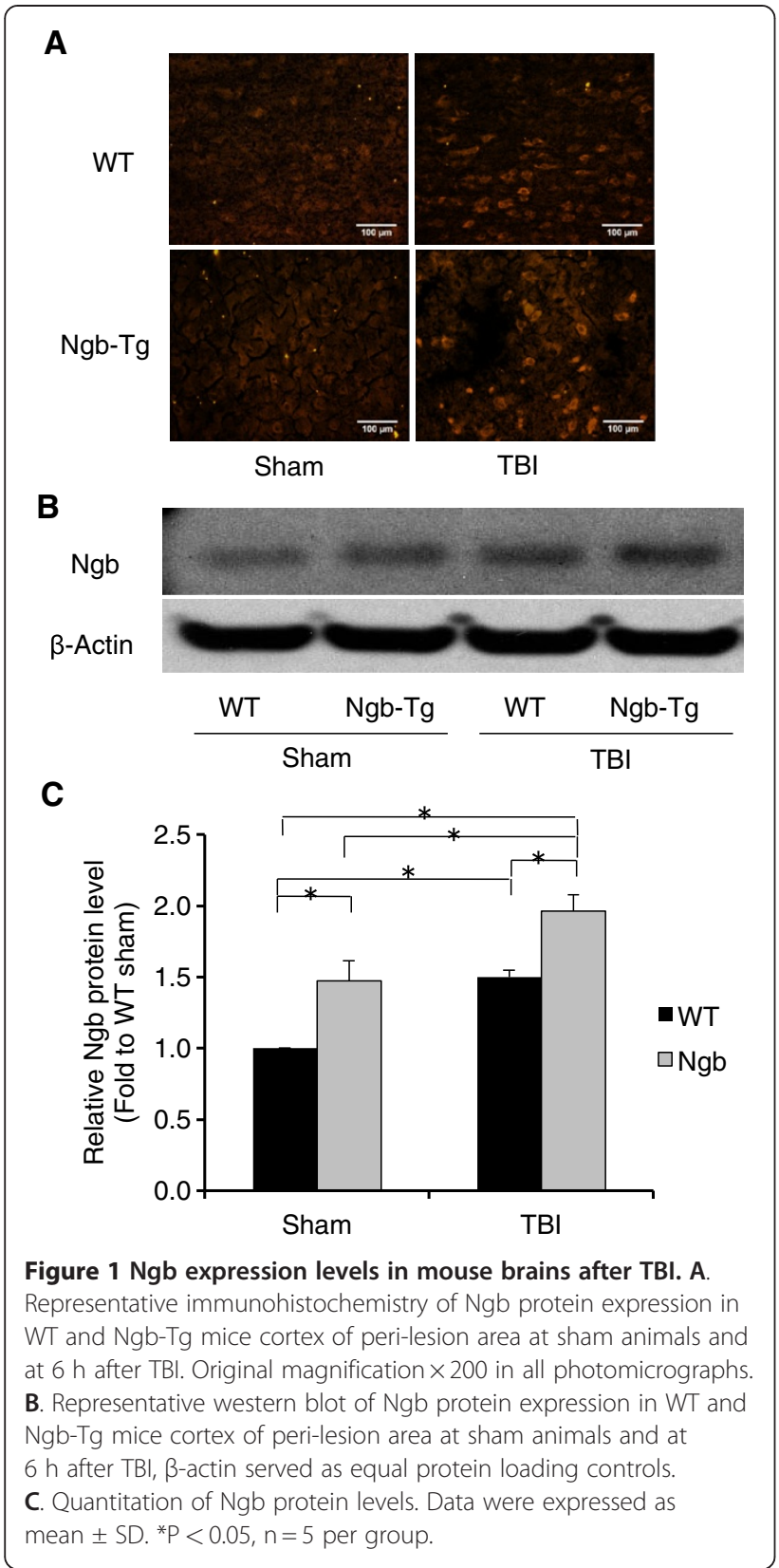

three weeks after TBI. At 0,1, 3, 5, 7, 10, 14, 21 days after TBI, sensorimotor functions were assessed by neurological score and hanging wire tests. Body weight loss was also measured on each test day. Experimental data showed significant deficits in all tests from day 1 to day 7 after TBI. All deficits were recovered close to preinjury baselines by day 21 after CCI. However, there were no statistically significant differences between NgbTg and WT mice in all assessments during the 3-week TBI recovery period (Table 1 ). We also used Morris Water Maze to assess spatial memory acquisition at 15-21 days after TBI, but we did not find significant differences between Ngb-Tg and WT mice in latency for hidden and visible platform trials (Figure $3 \mathrm{~A}$ ), as well as in probe trials (Figure 3B). Lastly, we quantitatively examined traumatic brain lesion volume and found it was significantly reduced in Ngb-Tg mice compared to WT mice at 21 days after TBI (Figure 4).

In the past two decades neuroprotectants that seek to block or inhibit one specific step in the cascades of TBI have not been very clinically successful [24]. It has been shown that TBI triggers endogenous protective mechanisms that can prevent or limit brain damage. Methods that seek to augment the brain's own endogenous protection and repair signals may lead to new therapeutic strategies for stroke and related disorders [25]. Experimental studies from our lab and others have documented that Ngb is one of very few unique molecules for endogenous neuroprotection, which functions in stabilizing neuronal function and prosurvival genes under both normal rest and hypoxic/ischemic conditions, serving to protect against oxidative stress and preserve mitochondrial function $[4,9,26]$. Furthermore, investigators have recently been attempting to elucidate Ngb gene regulation mechanisms, identifying small molecules that can specifically up-regulate endogenous Ngb protein expression for the development of novel endogenous neuroprotection strategies for treating neurological disorders $[19,27]$. Thus in this study, for the first time, we tested whether Ngb overexpression has neuroprotective effects in TBI model of mice. Results from present work showed: (1) TBI significantly increased Ngb expression in peri-lesion of ipsilateral cortex at $6 \mathrm{~h}$ after TBI compared to sham in both WT and Ngb-Tg mice, but the increased Ngb level was significantly higher in

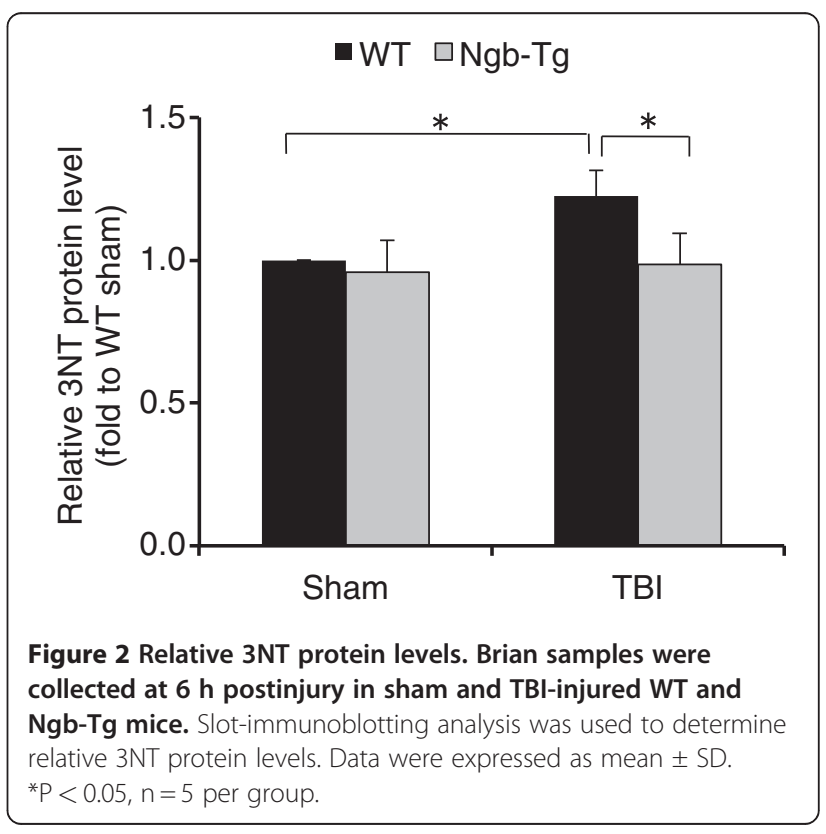


Table 1 Sensorimotor Function Assessments

\begin{tabular}{|c|c|c|c|c|c|c|}
\hline \multirow{2}{*}{$\begin{array}{l}\text { Tests } \\
\text { Group }\end{array}$} & \multicolumn{2}{|c|}{$\begin{array}{l}\text { Neurological score median } \\
\text { (25th to } 75 \text { th percentiles) }\end{array}$} & \multicolumn{2}{|c|}{$\begin{array}{l}\text { Hanging wire median } \\
\text { (25th to 75th percentiles) }\end{array}$} & \multicolumn{2}{|c|}{$\begin{array}{l}\text { Body Weight, } \\
\% \text { of day } 0\end{array}$} \\
\hline & WT & $\mathrm{Ngb}-\mathrm{Tg}$ & WT & Ngb-Tg & WT & Ngb-Tg \\
\hline Day 0 & 0 & 0 & $5.0(4.8-5.0)$ & $4.7(4.9-5.0)$ & 100 & 100 \\
\hline Day 1 & $3.0(2.0-3.5)$ & $5.0(3.0-6.0)$ & $3.0(2.2-3.7)$ & $3.0(2.0-3.3)$ & $97.2 \pm 6.67$ & $95.5 \pm 3.74$ \\
\hline Day 3 & $2.0(1.0-2.5)$ & $2.0(1.0-3.5)$ & $4.0(2.7-4.3)$ & $2.7(2.2-4.2)$ & $100.1 \pm 6.02$ & $97.5 \pm 2.18$ \\
\hline Day 5 & $1.0(1.0-2.0)$ & $2.0(1.0-2.0)$ & $4.0(3.3-4.7)$ & $4.0(3.2-4.3)$ & $99.7 \pm 6.81$ & $98.5 \pm 2.94$ \\
\hline Day 7 & $1.0(1.0-2.0)$ & $1.0(1.0-1.0)$ & $4.3(4.2-4.7)$ & $4.0(3.2-4.1)$ & $103.8 \pm 6.96$ & $97.8 \pm 4.09$ \\
\hline Day 10 & $1.0(1.0-2.0)$ & $0.0(0.0-1.0)$ & $4.3(4.0-4.8)$ & $4.0(4.0-4.0)$ & $103.8 \pm 6.96$ & $97.8 \pm 4.09$ \\
\hline Day 14 & $1.0(1.0-1.0)$ & $0.0(0.0-1.0)$ & $4.3(4.0-4.8)$ & $4.0(3.8-4.0)$ & $105.1 \pm 6.74$ & $99.4 \pm 4.32$ \\
\hline Day 21 & $1.0(0.0-1.0)$ & $1.0(0.0-1.0)$ & $4.3(4.1-5.0)$ & $4.0(3.8-4.0)$ & $108.3 \pm 7.48$ & $109.5 \pm 4.36$ \\
\hline
\end{tabular}

Sensorimotor deficits and body weight loss were assessed before (day 0) and at days 1, 3, 5, 7, 10, 14, 21 after CCl. Results are expressed as median values and $25^{\text {th }}$ to $75^{\text {th }}$ percentiles for assessments of neurological score, hanging wire tests; and mean \pm SD for assessments of body weight loss. $\mathrm{N}=11$ for Ngb-Tg and 15 for WT per group. In all assessments, there were no statistical significant differences between WT and Ngb-Tg groups.

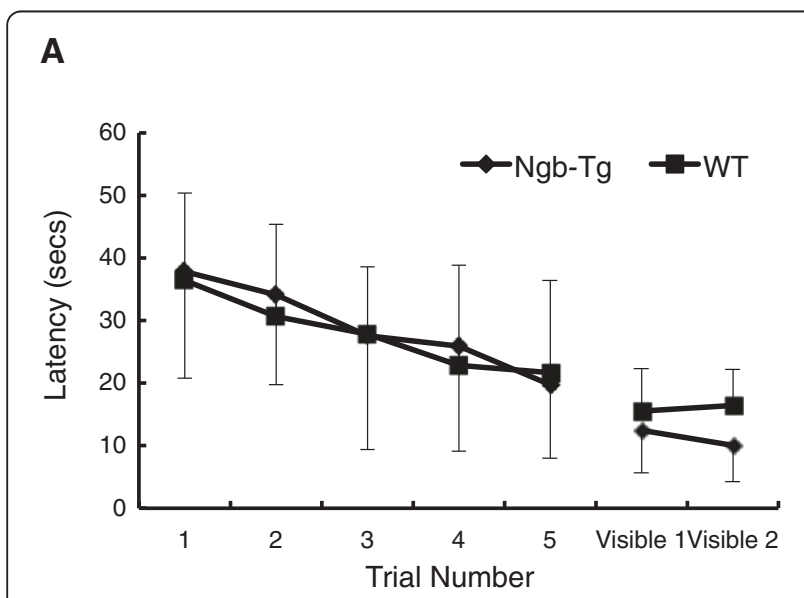

B

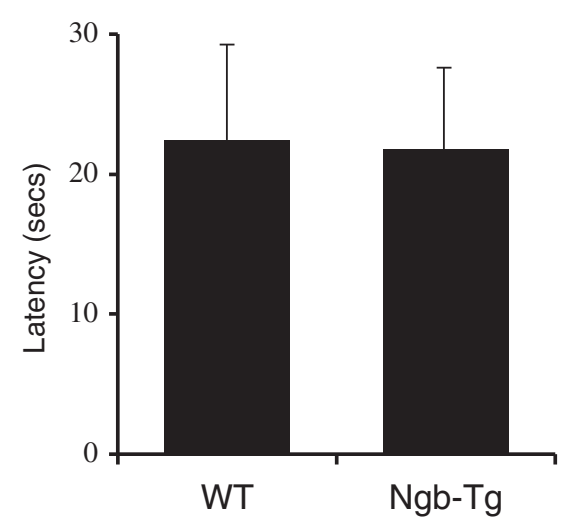

Figure 3 Spatial memory acquisitions in WT and Ngb-Tg mice after TBI. A. Morris water maze latencies were measured on days 15-21 for hidden and visible platform trials after TBI. B. Morris water maze latencies were measured on days 15-21 for probe trials after TBI. Data were expressed as mean \pm SD. $n=15$ for WT and 11 for Ngb-Tg per group, respectively.
Ngb-Tg mice than WT controls; (2) Ngb-Tg significantly reduced the levels of oxidative damage marker $3 \mathrm{NT}$ at $6 \mathrm{~h}$ after TBI in Ngb-Tg mice compared to WT controls; (3) Ngb-Tg mice exhibited smaller lesion volumes at 3 weeks after TBI compared to WT; (4) however, there were no significant differences in neurobehavioral deficits between the WT and Ngb-Tg mice after TBI for up to three weeks.

Ngb-Tg mice exhibited smaller cortical lesion volumes, suggesting that Ngb overexpression may prevent TBIinduced brain tissue damage. Oxidative stress has a mechanistic role in the pathophysiology of many neurologic diseases, including traumatic brain injury [15], and previous studies have shown that Ngb over-expression may reduce hypoxia/ischemia-induced oxidative stress in cultured neurons and a focal stroke mouse model $[4,5,9]$. Additionally, $3 \mathrm{NT}$ production at early time points after brain injury is associated with the degrees of TBI-induced oxidative tissue damage [15,28,29]. Thus, we tested and compared 3-NT production levels in the brain tissues of Ngb-Tg mice and WT mice after TBI. The significant reduction of 3-NT production in Ngb-Tg mouse brains suggested that Ngb over-expression may protect TBI brain damage, at least partially, by decreasing oxidative stress, which has been similarly observed in other studies of cells cultures and animal disease models $[5,9,19]$. Although we did know exactly why there was no effects of Ngb-Tg in oxidative stress reduction in the sham mice, but we may speculate that Ngb may have multiple functions for cell protection by maintaining hemostasis via stress sensing, or transducing signals or direct action. Ngb might respond stress to eliminate excessively elevated oxidative signaling and tissue damage, but is not or less effectively taking action under physiological resting conditions [4,8-11]. 


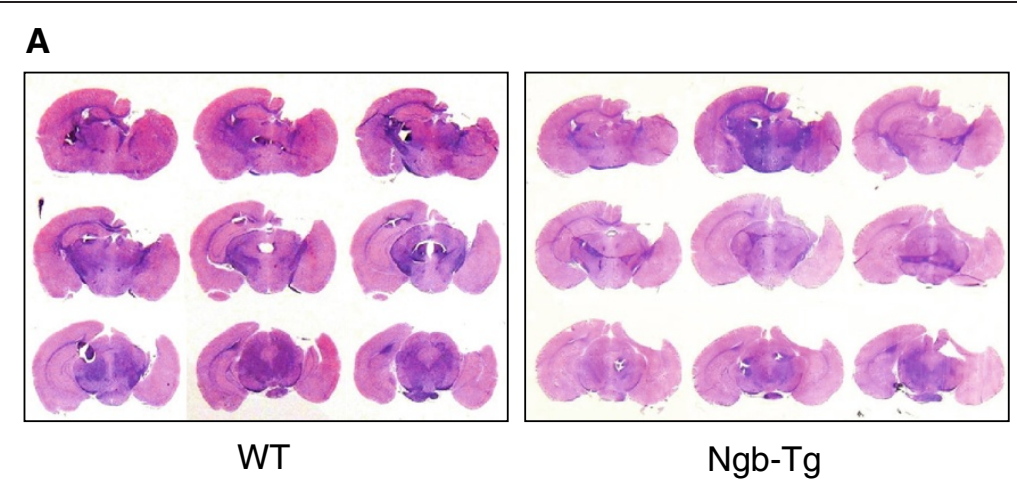

B

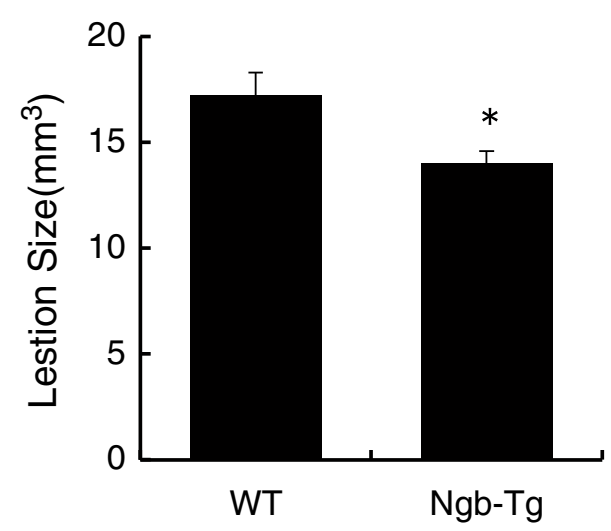

Figure 4 Measurements of cortical lesion volume in WT and Ngb-Tg mice. A. Representative photomicrographs of the traumatic lesions in H\&E stained WT and Ngb-Tg mouse brain sections at 21 days after TBI. B. Traumatic brain lesion size. Data were expressed as mean \pm SD. ${ }^{*} \mathrm{P}<0.05, \mathrm{n}=15$ for $\mathrm{WT}$ and 11 for Ngb-Tg per group, respectively.

Therefore the basal level of $3 \mathrm{NT}$ in sham group was not significantly altered as observed in present work.

We acknowledge that the early reduction of $3 \mathrm{NT}$ production at 6 hours after TBI in Ngb-Tg mice might be correlated with the lesion reduction at 3 weeks, but other factors likely also contribute to the neuroprotection of Ngb-Tg in TBI. A number of laboratories, including ours, have demonstrated that Ngb is protective against hypoxic/ischemic brain injury. Although the underlying mechanisms remain poorly defined, initial evidence suggests that the neuroprotective effect of Ngb may be linked largely to the structural features related to $\mathrm{O}_{2}$ and NO binding. Furthermore, putative signal transduction and mitochondrial function preservation also may be involved in the protective mechanisms $[4,8-11]$. However the involved molecular mechanisms need to be investigated in further studies.

It is worthy of noting that, there are two published studies closely related to the present work. A recent study showed that over-expression of Ngb in cultured neurons was neuroprotective against mechanical injury in vitro. This study also showed increased Ngb expression levels with peak time of 6 hours after TBI, and these increased levels correlated to the severity of TBI in rats [23]. Thus, we picked only the time point of 6 hours after TBI for examining changes of Ngb expression and 3NT production after TBI in present study. One of our previous studies in focal stroke showed oxidative tissue damage biomarker malondialdehyde levels in ischemic hemispheres of $\mathrm{Ngb}-\mathrm{Tg}$ were significantly reduced compared with wild-type controls at 8 hours and 22 hours after transient focal cerebral ischemia. Brain infarction volumes 1 day and 14 days after stroke were significantly reduced in Ngb-Tg mice. However, there were no significant detectable improvements in sensorimotor deficits for up to 14 days after stroke in Ngb-Tg mice compared with wild-type controls [5], which was similarly observed in present work in TBI mice.

We are aware that there are a few limitations in this study. First, the total Ngb protein expression levels in our Ngb-Tg mouse line is about 1.5 fold of WT control, which is relatively lower than when the Ngb-Tg mouse line was newly generated 5 years ago [5]. The decline of the Ngb protein level in Ngb-Tg mice may be mainly due to the endogenous depletion of inserted exogenous DNA fragments or inactivation of its promoter [30,31]. 
However findings from present work are still very informative and suggest that Ngb over-expression might be a new target for TBI therapy strategy development. We are working on a generation of neuron-specific and inducible Ngb-Tg mouse line for future studies of Ngb over-expression effects in TBI neurological outcomes and underlying molecular mechanisms. The second limitation is related to mechanisms. We only detected the reduction of 3-NT production in the Ngb-Tg mouse brains at the early time of 6 hours after TBI, suggesting there might be an antioxidative role for Ngb in vivo. However, for in vivo TBI models, it is difficult to unequivocally prove causality because reduced tissue damage may secondarily contribute to decreased oxidative stress and/or mitochondrial dysfunction. The third limitation is that we failed to detect significant differences in sensorimotor function or spatial memory function recovery between WT and Ngb-Tg mice. In part, this is because the TBI setting in our study might not be severe enough to cause long-term sensorimotor deficits since most of the deficits recovered close to the pre-injury baseline from one to two weeks after TBI. Also, the Ngb protein level in Ngb-Tg mouse brain might not be high enough. Alternatively, we may have simply lacked power and larger numbers of test animals would have helped. Further investigation is warranted to carefully define the role of Ngb in both long-term sensorimotor and cognitive deficits after TBI.

\section{Conclusions}

These experimental results suggest that Ngb overexpression can reduce traumatic brain lesion volume, possibly through reduction of oxidative stress.

\section{Abbreviations}

3NT: 3-nitrotyrosine; CCl: Controlled cortical impact; Ngb: Neuroglobin; NgbTg: Neuroglobin over-expression transgenic; NSS: Neurological severity score; PBS: Phosphate buffered saline; TBI: Traumatic brain injury; TBS: Tris buffered saline; WT: Wild type.

\section{Authors' contributions}

SZ: Performed TBI model and immunohistochemistry, drafted manuscript. ZY: Performed behavior tests and draft manuscript. GZ: Helped in experimental design. CX: Performed western blots. KH: Performed immunohistochemistry. MJW: Helped in experimental design and performed TBI model. JML: Performed behavior tests and data analysis. EHL: Helped in experimental design and troubleshooting. XW: Designed experiments, analyzed data, drafted and finalized manuscript. All authors read and approved the final manuscript.

\section{Acknowledgments}

This work was supported in part by NIH grant R01-NS049476 (to X.W.). We appreciate Ms. Jessica L. Poppe for language editorial assistance.

\section{Author details}

${ }^{1}$ Departments of Orthopedic and Neurosurgery, The First Bethune Hospital of Jilin University, Changchun, Jilin, China. ${ }^{2}$ Neuroprotection Research Laboratory, Departments of Neurology and Radiology, Massachusetts General Hospital, Harvard Medical School, Charlestown, MA, USA. ${ }^{3}$ Department of Pediatrics, Pediatric Critical Care Medicine, Massachusetts General Hospital, Harvard Medical School, Charlestown, MA, USA. ${ }^{4}$ Neuroprotection Research
Laboratory, Departments of Neurology and Radiology, Massachusetts General Hospital, 149 13th Street, Room 2401, Charlestown, MA 02129, USA.

Received: 21 March 2012 Accepted: 24 May 2012

Published: 15 June 2012

\section{References}

1. Awenius C, Hankeln T, Burmester T: Neuroglobins from the zebrafish Danio rerio and the pufferfish Tetraodon nigroviridis. Biochem Biophys Res Commun 2001, 287(2):418-421.

2. Burmester $T$, Weich $B$, Reinhardt $S$, Hankeln $T$ : A vertebrate globin expressed in the brain. Nature 2000, 407(6803):520-523.

3. Burmester T, Hankeln T: Neuroglobin: a respiratory protein of the nervous system. News Physiol Sci 2004, 19:110-113.

4. Liu J, Yu Z, Guo S, Lee SR, Xing C, Zhang C, Gao Y, Nicholls DG, Lo EH, Wang $X$ : Effects of neuroglobin overexpression on mitochondrial function and oxidative stress following hypoxia/reoxygenation in cultured neurons. J Neurosci Res 2009, 87(1):164-170.

5. Wang X, Liu J, Zhu H, Tejima E, Tsuji K, Murata Y, Atochin DN, Huang PL, Zhang C, Lo EH: Effects of neuroglobin overexpression on acute brain injury and long-term outcomes after focal cerebral ischemia. Stroke 2008, 39(6):1869-1874.

6. Khan AA, Wang Y, Sun Y, Mao XO, Xie L, Miles E, Graboski J, Chen S, Ellerby $L M$, Jin $K$, et al: Neuroglobin-overexpressing transgenic mice are resistant to cerebral and myocardial ischemia. Proc Natl Acad Sci USA 2006, 103 (47):17944-17948.

7. Khan AA, Mao XO, Banwait S, Jin K, Greenberg DA: Neuroglobin attenuates beta-amyloid neurotoxicity in vitro and transgenic Alzheimer phenotype in vivo. Proc Natl Acad Sci USA 2007, 104(48):19114-19119.

8. Yu Z, Liu J, Guo S, Xing C, Fan X, Ning M, Yuan JC, Lo EH, Wang X: Neuroglobin-overexpression alters hypoxic response gene expression in primary neuron culture following oxygen glucose deprivation. Neuroscience 2009, 162(2):396-403.

9. Jin K, Mao XO, Xie L, Khan AA, Greenberg DA: Neuroglobin protects against nitric oxide toxicity. Neurosci Lett 2008, 430(2):135-137.

10. Khan AA, Mao XO, Banwait S, DerMardirossian CM, Bokoch GM, Jin K, Greenberg DA: Regulation of hypoxic neuronal death signaling by neuroglobin. FASEB J 2008, 22(6):1737-1747.

11. Yu Z, Fan $X$, Lo EH, Wang $X$ : Neuroprotective roles and mechanisms of neuroglobin. Neurol Res 2009, 31(2):122-127.

12. Wang X, Jung J, Asahi M, Chwang W, Russo L, Moskowitz MA, Dixon CE, Fini $M E$, Lo EH: Effects of matrix metalloproteinase-9 gene knock-out on morphological and motor outcomes after traumatic brain injury. J Neurosci 2000, 20(18):7037-7042.

13. Lok J, Leung W, Zhao S, Pallast S, van Leyen K, Guo S, Wang X, Yalcin A, Lo EH: gamma-glutamylcysteine ethyl ester protects cerebral endothelial cells during injury and decreases blood-brain barrier permeability after experimental brain trauma. J Neurochem 2011, 118(2):248-255.

14. Sultana R, Butterfield DA: Slot-blot analysis of 3-nitrotyrosine-modified brain proteins. Methods Enzymol 2008, 440:309-316.

15. Deng Y, Thompson BM, Gao X, Hall ED: Temporal relationship of peroxynitrite-induced oxidative damage, calpain-mediated cytoskeletal degradation and neurodegeneration after traumatic brain injury. Exp Neurol 2007, 205(1):154-165.

16. Tsenter J, Beni-Adani L, Assaf $Y$, Alexandrovich AG, Trembovler V, Shohami E: Dynamic changes in the recovery after traumatic brain injury in mice: effect of injury severity on T2-weighted MRI abnormalities, and motor and cognitive functions. J Neurotrauma 2008, 25(4):324-333.

17. Beni-Adani L, Gozes I, Cohen $Y$, Assaf $Y$, Steingart RA, Brenneman DE, Eizenberg $O$, Trembolver $V$, Shohami E: A peptide derived from activity-dependent neuroprotective protein (ADNP) ameliorates injury response in closed head injury in mice. J Pharmacol Exp Ther 2001, 296(1):57-63.

18. Bermpohl D, You Z, Korsmeyer SJ, Moskowitz MA, Whalen MJ: Traumatic brain injury in mice deficient in Bid: effects on histopathology and functional outcome. J Cereb Blood Flow Metab 2006, 26(5):625-633.

19. Liu N, Yu Z, Xiang S, Zhao S, Tjarnlund-Wolf A, Xing C, Zhang J, Wang X: Transcriptional regulation mechanisms of hypoxia-induced neuroglobin gene expression. Biochem J 2012, 443(1):153-164.

20. Sun Y, Jin K, Peel A, Mao XO, Xie L, Greenberg DA: Neuroglobin protects the brain from experimental stroke in vivo. Proc Natl Acad Sci USA 2003, 100(6):3497-3500. 
21. Lin X, Li M, Hu YZ, Han ZT, Zhang HH, Shang AJ, Gao DW, Zhou DB: An experimental research of neuroglobin expression changes and neural apoptosis after traumatic brain injury. Zhongguo Ying Yong Sheng Li Xue Za Zhi 2010, 26(1):39-44.

22. Chuang PY, Conley YP, Poloyac SM, Okonkwo DO, Ren D, Sherwood PR, Hravnak M, Alexander SA: Neuroglobin genetic polymorphisms and their relationship to functional outcomes after traumatic brain injury. J Neurotrauma 2010, 27(6):999-1006.

23. Shang A, Liu K, Wang H, Wang J, Hang X, Yang Y, Wang Z, Zhang C, Zhou D: Neuroprotective effects of neuroglobin after mechanical injury. Neurol Sci 2012, 33(3):551-558.

24. Jain KK: Neuroprotection in traumatic brain injury. Drug Discov Today 2008, 13(23-24):1082-1089.

25. Dirnagl U, Simon RP, Hallenbeck JM: Ischemic tolerance and endogenous neuroprotection. Trends Neurosci 2003, 26(5):248-254.

26. Wei X, Yu Z, Cho KS, Chen H, Malik MT, Chen X, Lo EH, Wang X, Chen DF: Neuroglobin is an endogenous neuroprotectant for retinal ganglion cells against glaucomatous damage. Am J Pathol 2011, 179(6):2788-2797.

27. Jin $K$, Mao XO, Xie L, John V, Greenberg DA: Pharmacological induction of neuroglobin expression. Pharmacology 2011, 87(1-2):81-84.

28. Ansari MA, Roberts KN, Scheff SW: Oxidative stress and modification of synaptic proteins in hippocampus after traumatic brain injury. Free Radic Biol Med 2008, 45(4):443-452.

29. Hall ED, Detloff MR, Johnson K, Kupina NC: Peroxynitrite-mediated protein nitration and lipid peroxidation in a mouse model of traumatic brain injury. J Neurotrauma 2004, 21(1):9-20.

30. Houdebine LM: The methods to generate transgenic animals and to control transgene expression. J Biotechnol 2002, 98(2-3):145-160.

31. Sijen T, Vijn I, Rebocho A, van Blokland R, Roelofs D, Mol JN, Kooter JM: Transcriptional and posttranscriptional gene silencing are mechanistically related. Curr Biol 2001, 11(6):436-440.

doi:10.1186/1471-2202-13-67

Cite this article as: Zhao et al:: Neuroglobin-overexpression reduces traumatic brain lesion size in mice. BMC Neuroscience 2012 13:67.

\section{Submit your next manuscript to BioMed Central and take full advantage of:}

- Convenient online submission

- Thorough peer review

- No space constraints or color figure charges

- Immediate publication on acceptance

- Inclusion in PubMed, CAS, Scopus and Google Scholar

- Research which is freely available for redistribution 